\title{
Scripted Reality-Sendungen
}

\author{
Studienbeispiele zur gezeigten Medienrealität sowie \\ Rezeption und Wirkung von Scripted Reality-Sendungen
}

von Dr. Hanna Gölz-Weis

\section{Die Welt des Realitätsfernsehens}

Die Frage nach negativen Wirkungen des sogenannten Realitätsfernsehens (Reality-TV) steht seit nahezu 30 Jahren immer wieder auf der Agenda verschiedenster Gruppen und Verbänden (vgl. Gölz \& Niemann, 2015). So gibt es Befürchtungen, dass sich die Zuschauer an den gezeigten Inhalten ergötzen oder gar die gezeigte Medienrealität übernehmen könnten (so genannte Kultivierungseffekte). In der Kultivierungsforschung geht man davon aus, dass eine im Vergleich zur Realität verzerrte Medienrealität zu einer verzerrten Realitätswahrnehmung führt. Dabei wird zwischen Effekten erster Ordnung (verzerrte Einschätzung von Häufigkeitsverteilungen) und Effekten zweiter Ordnung (Veränderung von Einstellungen und Wertvorstellungen) unterschieden (vgl. im Überblick Meltzer, 2019). Der Forschungsstand zu Kultivierungseffekten durch das Realitätsfernsehen ist allerdings durchwachsen. Es gibt nur wenige Studien, die sich den Formaten innerhalb des Reality-TV sowie den gezeigten Inhalten im Detail annehmen. Gleichzeitig lassen sich langfristige Effekte auf Seiten der Rezipienten nur schwer nachweisen. Erschwerend kommt hinzu, dass unter der Genrefamilie Reality- $T V$ viele verschiedene Genres zusammengefasst und Begriffe nicht einheitlich verwendet werden (vgl. Gölz \& Niemann, 2015)

\section{Verortung von Scripted Reality-Formaten im Bereich des Realitätsfernsehens}

Realitätsfernsehen kann in zwei Formen (Klaus \& Lücke, 2003) unterschieden werden: Das performative Realitätsfernsehen bietet eine Bühne für nicht Alltägliches und greift direkt in die Alltagswirklichkeit der Teilnehmer ein. Ein typisches Beispiel für diese Formate sind Castingshows („Germany's Next Topmodel“, ProSieben) oder auch Beziehungsshows („Bauer sucht Frau“, RTL). Das narrative Realitätsfernsehen hingegen stellt realitätsnahe Ereignisse nicht prominenter Laien-Darsteller authentisch nach und erzeugt aufgrund formaler Stilmittel eine hohe Authentizität. Darunter fallen auch Scripted Reality-Formate wie bspw. „Anwälte im Einsatz“ (Sat.1), „Berlin Tag \& Nacht“ (RTL II) oder „Hilf mir! Jung, pleite, verzweifelt...“ (RTL II).

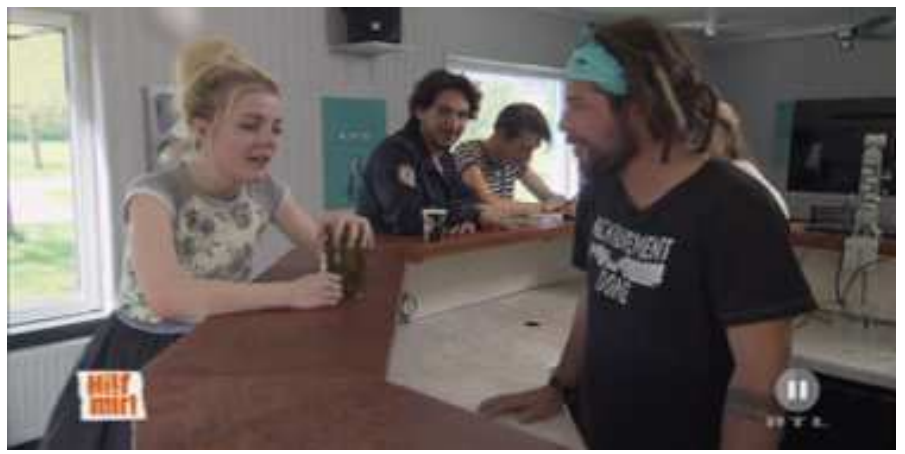

Innerhalb der Scripted Reality-Formate kann noch einmal in episodische (abgeschlossene) und soapartige (andauernde) Formate unterschieden werden. Während es bei den soapartigen Formaten einen festen Cast gibt und sich die Handlungsstränge und Geschichten über mehrere Episoden weiterentwickeln („Berlin Tag \& Nacht“, RTL II), werden die Handlungsstränge bei den episodischen Formaten („Hilf mir! Jung, pleite, verzweifelt...", RTL II) innerhalb jeder Episode abgeschlossen und es gibt wenn überhaupt nur, einzelne feste Experten

\section{Gezeigte Wirklichkeit und Rezeption}

(vgl. Schenk, Gölz \& Niemann 2015).

Zwei Studien beleuchten die in diesen Formaten gezeigte Medienrealität ausführlich: In der Studie Fernsehrealität und Realitätswahrnehmung: Eine Untersuchung zum Einfluss von Scripted-Reality-Sendungen auf Erwachsene (Gölz, 2018) werden neben der Akteurs- und Sendungsebene besonders die gezeigten Familien betrachtet.

Eine Medieninhaltsanalyse von 120 Folgen aus sechs episodischen Formaten im Jahr 2014 zeigte, dass formale Stilmittel sowie gezeigte Inhalte und Akteure eine hohe Realitätsnähe erzeugen. In diesen Formaten mit häufig gleichbleibenden thematischen Schwerpunkten wird eine Welt gezeigt, die voller Probleme ist, welche gleichzeitig aber auch immer in einem Happy End münden. Dies bestätigt ältere Ergebnisse von Schenk, Gölz und Niemann (2015).

In einer nachgelagerten quantitativen Studie zu episodischen Scripted Reality-Formaten wurden Erwachsene zu Nutzungsmotiven, Einordnung der gezeigten Fernsehrealität, persönlichen Werten sowie Kultivierungsaspekten befragt (Gölz, 2018). Als zentrale Ergebnisse konnten die Nutzungsmotive Unterhaltung und Zeitvertreib sowie ein Zusammenhang zwischen der Nutzung und den persönlichen Werten der $\mathrm{Zu}-$ schauer (bspw. Materialismus) identifiziert werden. Weiterhin zeigte sich ein positiver Zusammenhang zwischen der Einordnung der Inhalte als non-fiktionale und in sich konsistente Geschichte sowie dem Alter der Befragten. Ein negativer Zusammenhang zwischen der zugesprochenen Realitätsnähe und der Bildung macht deutlich: je niedriger die formale Bildung der Befragten war, desto realitätsnäher schätzten sie das Gezeigte ein. Darüber hinaus zeigten sich Kultivierungseffekte hinsichtlich einer Schlechte Welt- und einer Heile Welt-Sicht, welche von der zugesprochenen Realitätsnähe bzw. der zugesprochenen Stimmigkeit der Geschichte (= narrative Konsistenz) mit bestimmt wird. Das Erkennen des Inszenierungscharakters des Gezeigten hat dabei keinerlei Auswirkungen auf diese Effekte.

In einer anderen Studie zeigten sich bei Jugendlichen ebenfalls Kultivierungseffekte, die durch das Nichterkennen der Inszenierung unterstützt werden. Insbesondere Jüngere mit einer formal niedrigeren Bildung durchschauen den Inszenierungscharakter oftmals nicht, diese Zielgruppe wird vermutlich durch die dargestellte Medienrealität im Besonderen (negativ) beeinflusst (Schenk, Gölz \& Niemann, 2015). 
Arbeitsgemeinschaft der Landesmedienanstalten in der Bundesrepublik Deutschland (Hrsg.). (2013). Programmbericht 2012: Fernsehen in Deutschland. Berlin: Vistas-Verlag.

Lünenborg, M., Martens, D., Köhler, T., \& Töpper, C. (2011). Skandalisierung im Fernsehen: Strategien, Erscheinungsformen und Rezeption von Reality-TV-Formaten. Schriftenreihe Medienforschung der Landesanstalt für Medien NordrheinWestfalen: Bd. 65. Berlin: Vistas-Verlag.

Mikos, L. (2012): Das Spiel mit der Realität. Darstellungsformen im Reality TV. In: Televizion 25 (1), S. 48-51.
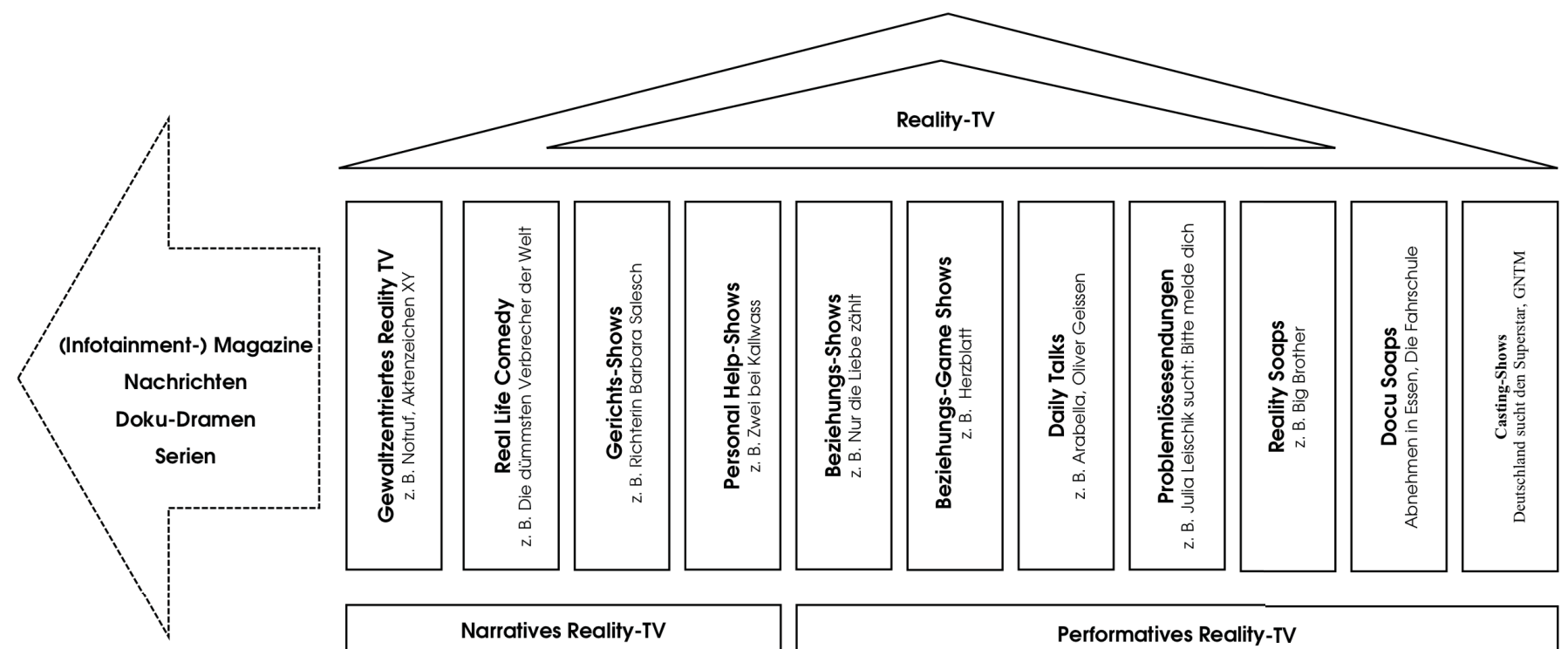

Narratives Reality-TV

Performatives Reality-TV

\begin{tabular}{lllll}
\hline $\begin{array}{l}\text { Verwandtschaft mit } \\
\text { anderen Gattungen }\end{array}$ & Talk-Show & Game Show & & Dokumentation \\
& & Fernseh-Show & & Soap Opera \\
\hline
\end{tabular}

Abbildung 2: Formen des Reality-TV (in Anlehnung an Klaus und Lücke, 2003, S. 200)

\section{Schlussfolgerungen}

Scripted Reality-Formate zeichnen sich durch eine besonders hohe emotionale Erlebnisqualität, eine leichte Verständlichkeit sowie durch die Art der Darstellung aus. Dies mündet in einem hohen Identifikationspotenzial für die Zuschauer. Gleichzeitig werden (zwischenmenschliche) Konflikte in einer stark vereinfachten Form gelöst. Damit bilden diese Formate allerdings keinen Einzelfall in der Fernsehlandschaft. Die Medienkompetenz von Rezipienten spielt für das Erkennen der medialen Inszenierungen eine wichtige Rolle, da diese Formate den Zuschauern auch Wissen zur Selbst-Organisation oder zur Selbst-Reflektion an die Hand gibt (Bandura, 2001, S. 266). Zur Herausbildung von Medienkompetenz in diesem Berech ist eine begleitete Rezeption dieser Inhalte sinnvoll - ein Verbot bestimmter Inhalte oder Formate stellt keine zielführende Handlungsoption dar. An dieser Stelle können auch weitergehende medienpädagogische Angebote ansetzen. Zum Beispiel indem zirkuläre Prozesse einer zwischenmenschlichen Konfliktaushandlung thematisiert und anhand von Fallbeispielen und Rollenspielen vertieft werden. Eine Option ist auch die begleitete Rezeption, wobei die gezeigten Medieninhalte kritisch reflektiert und eingeordnet werden müssen. Die Kultivierung von Weltbildern ist aus medienpädagogischer Sicht ebenso als durchaus problematisch einzuordnen. Entsprechend ist es wichtig, dass Rezipienten soziale Umgangsformen und soziale Beziehungen aus der Realität kennen und verstehen lernen.

Auf Grund der Kurzlebigkeit des Genres bzw. der ganzer Formate sollten solche Überlegungen genreübergreifend stattfinden und eine eher

\section{Autorin der Ausgabe}

Dr. Hanna Gölz-Weis ist Laborleiterin des ZEEB an der Dualen Hochschule Baden-Württemberg (DHBW) in Mannheim. Kontakt: hanna.goelz-weis@dhbw-mannheim.de

\section{Empfohlene Zitation des Beitrages}

Gölz-Weis, H. (2019). Scripted-Reality-Sendungen. In: B. Bigl (Hrsg.). Transfer Pus - Beiträge zur Medienbildung. Issue 02/2019. https://nbn-resolving.org/urn:nbn:de:bsz: 14-qucosa2-344214 allgemeine Medienkompetenz im Mittelpunkt stehen als formatspezifische Überlegungen. Das heißt, statt der ausschließlichen Thematisierung von Scripted Reality-Sendungen sollte allgemein die gezeigte Medienrealität gezielt angesprochen und sowohl junge Rezipienten als auch Erwachsene gezielt eingebunden werden. Denn insbesondere Rezipienten mit einer formal niedrigeren Bildung haben Probleme bei der korrekten Einordnung der in Scripted Reality gezeigten Inhalte - egal welchen Alters. Außerdem hilft eine in der Schulzeit entwickelte, kritische Haltung bei der Einordnung etwaiger neuer Formate im Erwachsenenalter.

\section{Quellen}

Bandura, A. (2001). Social cognitive theory of mass communication.

Media Psychology, 3(3), S. 265-299. https://doi.org/10.1207/

S1532785XMEP0303 03

Gölz, H., \& Niemann, J. (2015). Zur Einführung des

Untersuchungsgegenstands der Scripted Reality-Formate. In M.

Schenk, H. Gölz, \& J. Niemann (Hrsg.), Faszination Scripted

Reality: Realitätsinszenierungen und deren Rezeption durch

Heranwachsende. Düsseldorf: Landesanstalt für Medien Nordrhein-

Westfalen (LfM), S. 34-75.

Meltzer, Christine E. (2019): Kultivierungsforschung. 1. Auflage. Baden-Baden: Nomos.

Klaus, E., \& Lücke, S. (2003). Reality TV - Definition und Merkmale einer erfolgreichen Genrefamilie am Beispiel von Reality Soap und Docu Soap. M\&K, 51(2), S. 195-212.

Schenk, M., Gölz, H., \& Niemann, J. (2015). Faszination Scripted Reality: Realitätsinszenierungen und deren Rezeption durch Heranwachsende. Düsseldorf: Landesanstalt für Medien NordrheinWestfalen (LfM).

Gölz, H. (2018). Fernsehrealität und Realitätswahrnehmung: Eine Untersuchung zum Einfluss von Scripted-Reality-Sendungen auf Erwachsene. Baden-Baden: Nomos. 